Acta Crystallographica Section A

Foundations of Crystallography

ISSN 0108-7673

Received 16 August 2002 Accepted 8 October 2002

(C) 2003 International Union of Crystallography Printed in Great Britain - all rights reserved

\section{Three-periodic nets and tilings: regular and quasiregular nets}

\author{
Olaf Delgado Friedrichs, ${ }^{a}+$ Michael $\mathrm{O}^{\prime}$ Keeffe $^{\mathrm{a} *}$ and Omar M. Yaghib \\ ${ }^{\mathbf{a}}$ Department of Chemistry, Arizona State University, Tempe, AZ 85287, USA, and ${ }^{\mathbf{b}}$ Department of \\ Chemistry, University of Michigan, Ann Arbor, MI 48109, USA. Correspondence e-mail: \\ mokeeffe@asu.edu
}

Regular nets are defined as those with symmetry that requires the coordination figure to be a regular polygon or polyhedron. It is shown that this definition leads to five regular 3-periodic nets. There is also one quasiregular net with a quasiregular coordination figure. The natural tiling of a net and its associated essential rings are also defined, and it is shown that the natural tilings of the regular nets have the property that there is just one kind of vertex, one kind of edge, one kind of ring and one kind of tile, i.e. transitivity 1111. The quasiregular net has two kinds of natural tile and transitivity 1112.

\section{Introduction}

There is considerable interest in the basic nets underlying the topology of periodic structures such as those of crystals and considerable effort has been devoted to their enumeration (e.g. Barrer \& Villiger, 1969; Wells, 1977, 1979; Chung et al., 1985; Smith, 1988; Akporiaye \& Price, 1989; O'Keeffe, 1991, 1992, 1995; O'Keeffe \& Brese, 1992; Andries \& Smith, 1994; Han \& Smith, 1994, 1999a,b; Treacy et al., 1997; Delgado Friedrichs et al., 1999; Delgado Friedrichs \& Huson, 2000). Recently, we have argued (O'Keeffe et al., 2000) that the most important of these, and the most plausible targets for designed synthesis, are the ones that have 'simple, high-symmetry' structures. In this paper, we quantify this concept by first identifying five regular 3-periodic structures, and then show how the consideration of nets as based on tilings leads to natural quantification of 'regularity'. We note that there is no general agreement even on the definition of the term 'net', and it is not always clear if reference is to a graph, or to its embedding in Euclidean space in which coordinates are assigned to vertices. Our treatment is informal and we defer a more rigorous mathematical treatment of some statements to a later publication.

The five regular (Platonic) polyhedra and their geometric properties have been known for millennia (Euclid of Alexandria, ca 300 B.C.). These structures can be considered as tilings of a closed surface, topologically equivalent to the surface of a sphere, by congruent regular polygons such that there is just one kind of vertex, edge and face (i.e. vertices etc. related by operations of a symmetry group). The corresponding tilings of the plane (two-dimensional Euclidean space) were described some hundreds of years ago by Kepler (1619). The three regular tilings of the plane are by triangles, squares and hexagons, respectively.

$\uparrow$ Present address: Department of Computer Science, University of Tübingen, D-72076 Tübingen, Germany.
If one adopts the definition of regularity in three dimensions that the tiles be regular polyhedra and that there is just one kind of vertex, face, edge and tile, the tiling of the 3 -sphere leads to the six regular four-dimensional polytopes (Coxeter, 1973). However, the only regular tiling of threedimensional Euclidean space, according to this definition, is the familiar tiling by cubes. Coxeter (1973) refers to this lack of riches as 'an unfortunate accident', however, we believe rather that it is the result of a too-stringent definition of regularity that places emphasis on the tile rather than on the net of vertices and edges carried by the tiling (its 1-skeleton). Accordingly, we propose a definition of regularity that focuses exclusively on the net and leads to the recognition of five regular nets; only after we have identified regular nets do we examine the tilings that carry them. We find that the tilings associated with nets also lead to a second definition of regularity that is in accord with the first.

The structures we describe here are all well known (see e.g. O'Keeffe \& Hyde, 1996). What is new is the recognition of regularity and the development of a hierarchical description of nets based in part on the concept of natural tilings. We will use this in subsequent papers to give a uniform treatment of the more-regular 3-periodic structures. We also propose a nomenclature (lower-case three-letter symbol) for these structures, as some have many names and symbols - for example our first structure is variously known as 'net (10,3)-a' (Wells, 1977), 'Laves net' (Pearce, 1978), ' $Y^{*}$ ' (Fischer \& Koch, 1983), '3/10/c1' (Koch \& Fischer, 1995), 'SrSi 2 net' (O'Keeffe et al., 2000), 'labyrinth graph of the gyroid surface' (Hyde \& Ramsden, 2000) - other structures have no names (that we are aware of) at all. The nomenclature is designed to parallel the widely accepted upper-case three-letter symbols used for zeolite frameworks (Baerlocher et al., 2001).

Most of the nets we consider can be realized as imbeddings in which edges are all of equal length and correspond to shortest intervertex distances. Such structures are called 
Table 1

Data for regular and quasiregular nets.

$\mathrm{lc}=$ lattice complex, $\mathrm{ps}=$ point symmetry, $\mathrm{sg}=$ space group, trans = transitivity. The last two rows are data for the fluorite (flu) structure.

\begin{tabular}{|c|c|c|c|c|c|c|c|c|}
\hline$Z$ & Coord. figure & lc & Name & ps & $\mathrm{sg}$ & $x y z$ & Tiles & trans \\
\hline 3 & Triangle & $Y^{*}$ & srs & 32 & $I 4_{1} 32$ & $1 / 8,1 / 8,1 / 8$ & {$\left[10^{3}\right]$} & 1111 \\
\hline 4 & Square & $J^{*}$ & nbo & $4 / \mathrm{mmm}$ & $\operatorname{Im} \overline{3} m$ & $0,1 / 2,1 / 2$ & {$\left[6^{8}\right]$} & 1111 \\
\hline 4 & Tetrahedron & $D$ & dia & $\overline{4} 3 m$ & $F d \overline{3} m$ & $1 / 8,1 / 8,1 / 8$ & {$\left[6^{4}\right]$} & 1111 \\
\hline 6 & Octahedron & $c P$ & pcu & $m \overline{3} m$ & $P m \overline{3} m$ & $0,0,0$ & {$\left[4^{6}\right]$} & 1111 \\
\hline 8 & Cube & $c I$ & bcu & $m \overline{3} m$ & $\operatorname{Im} \overline{3} m$ & $0,0,0$ & {$\left[4^{4}\right]$} & 1111 \\
\hline 12 & Cuboctahedron & $c F$ & feu & $m \overline{3} m$ & $F m \overline{3} m$ & $0,0,0$ & {$\left[3^{8}\right]+2\left[3^{4}\right]$} & 1112 \\
\hline 8 & Cube & & flu & $m \overline{3} m$ & $F m \overline{3} m$ & $0,0,0$ & {$\left[4^{12}\right]$} & 2111 \\
\hline 4 & Tetrahedron & & & $\overline{4} 3 m$ & & $1 / 4,1 / 4,1 / 4$ & & \\
\hline
\end{tabular}

The structures we have described are well known as the enantiomorphic pair of invariant lattice complexes ${ }^{+} Y^{*}$ and ${ }^{-} Y^{*}$. We mention also that all vertextransitive 3-coordinated sphere packings are known (Koch \& Fischer, 1995) and the $Y^{*}$ lattice complex is the only one with site symmetry (32) that includes a threefold axis. This net is found as the $\mathrm{Si}$ net of $\mathrm{SrSi}_{2}$ and for this reason we propose to use the name srs for this net.

(b) Square. As in case (a), one can have either a layer structure or a cubic structure and we consider only the latter. The vertices must be on fourfold axes

sphere packings and systematic enumerations of many of them have been given (Fischer, 1973, 1974, 1991a,b, 1993; Koch \& Fischer, 1995).

\section{Regular nets}

We consider only three-periodic nets with one kind of vertex (vertex transitive) and specifically refer to maximumsymmetry embeddings of the net. The coordination figure is defined by the convex hull of the neighbors of a vertex in this embedding, and we require this figure to be a regular polygon or polyhedron. We further require the vertex to have site symmetry in the net that is at least the rotation symmetry of that regular polygon or polyhedron. Note that we consider polygons to have a three-dimensional symmetry so that they must have at least the symmetry of the appropriate dihedral group (32 for a triangle, 422 for a square etc.). The symmetry requirement also means that the resulting structure has to correspond to an invariant lattice complex (atom coordinates fixed), and as these are well known (Fischer \& Koch, 1983) we have an independent check of our enumeration.

Taking into account the fact that the possible site symmetries must be a crystallographic point group, we can immediately state that the only possible coordination figures for a regular net are: $(a)$ equilateral triangle, $(b)$ square, $(c)$ regular plane hexagon, $(d)$ regular tetrahedron, $(e)$ regular octahedron, $(f)$ cube. We consider each case in turn.

(a) Equilateral triangle. With a threefold symmetry axis, the possibilities are either uniaxial symmetry, which can only result in a plane net with all coordination figures coplanar (which we reject as we are concerned only with three-periodic structures), or a cubic structure. Consider in the latter case one vertex, $A$, and its three neighboring vertices, $B, C, D$. The vertex figures associated with $B, C, D$ must be inclined at a dihedral angle equal to that of $A$ by the angle between cubic threefold axes, i.e. $\pm \cos ^{-1}(-1 / 3)= \pm 109.5^{\circ}$ as shown in Fig. 1. Note that the two possibilities are chiral. As $B, C$ and $D$ are on threefold axes, the orientation of the coordination figures of their neighbors (in each case $A$ and two others) are determined, and it should be clear that only two enantiomorphic structures are possible. and the coordination figures of neighbors must be at $90^{\circ}$ to the coordination figure of any given vertex. Fig. 3 shows that there is only one possibility and the resulting net is again familiar as the net of $\mathrm{NbO}$ or the lattice complex $J^{*}$. Our proposed name for this structure is nbo.

(c) Hexagon. Site symmetry with a sixfold axis as required for a regular plane hexagon is only possible for a planar structure, so we conclude that there is no regular 3-periodic structure with a hexagonal coordination figure. We note that there is a cubic structure with a regular hexagonal coordination figure. However, the site symmetry is $\overline{3} m$ and we prefer to consider this net as one of the larger class of semiregular nets discussed in the next paper in this series.

(d) Tetrahedron. The minimum symmetry for a regular tetrahedral coordination figure is 23 , so a regular tetrahedral structure must be cubic. Edges must be on threefold axes and therefore can only assume four distinct spatial directions. From this it is easy to see that neighboring vertices and the positions of their neighbors are fixed and must produce a unique structure which is in fact the familiar diamond structure, lattice complex $D$. We propose the name dia.

There are other invariant lattice complexes $\left(V, S^{*}, Q, W^{*}\right)$ with tetrahedral coordination, but it may quickly be verified that none other than $D$ has regular tetrahedral coordination.

(e) Octahedron. It is trivial to show that in this case the net of the primitive cubic lattice (symbol $c P$ ) is the only one with regular octahedral coordination. The proposed name is pcu.

(f) Cube. Likewise it is trivial to show that the net of the body-centered cubic lattice (symbol $c I$ ) is the only one with coordination figures that are regular cubes. We propose the name bcu for this net.

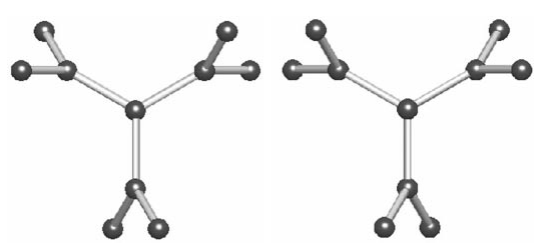

Figure 1

The two ways of propagating a three-dimensional 3-coordinated net with threefold symmetry at each vertex 
Various aspects of the regular nets are shown in Figs. 2-6. In the drawings of the nets, we actually show the augmented net (O'Keeffe et al., 2000) in which the vertices are replaced by a group of vertices having the conformation of the coordination figure. Table 1 summarizes some important properties of these structures.

\section{Quasiregular nets}

A quasiregular polyhedron is one in which all vertices and all edges are related by symmetry but which has two kinds of face. It is well known (Coxeter, 1973) that the only two possibilities are the cuboctahedron (3.4.3.4) with symmetry $m \overline{3} m$ and the icosidodecahedron (3.5.3.5) with symmetry $m \overline{3} \overline{5}$. A quasiregular net is defined as one in which the coordination figure is a quasiregular polyhedron. Clearly the cuboctahedron is the only possibility for a periodic structure and it is trivial to show that the only such net is that of the face-centered cubic lattice (symbol $c F$ ), proposed name fcu. This assignment is in accord with the identification by Coxeter (1973) of the facecentered cubic lattice as the only quasiregular tiling; i.e. one
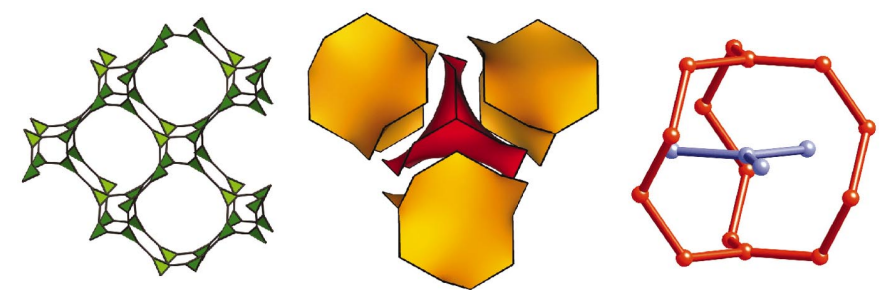

Figure 2

Left: the augmented $Y^{*}(\mathbf{s r s})$ net. Center; the tiling. Right: the skeleton of one tile (red) with a fragment of the dual net (blue).
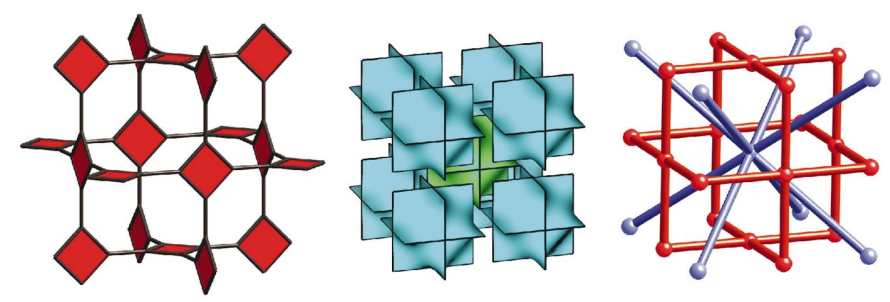

Figure 3

Left: the augmented NbO (nbo) net. Center: the tiling. Right: the skeleton of one tile (red) with a fragment of the dual net (blue).
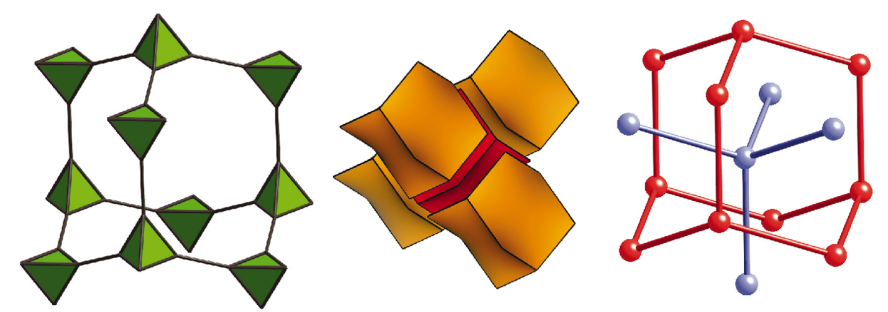

Figure 4

Left: the augmented diamond (dia) net. Center: the tiling. Right: the skeleton of one tile (red) with a fragment of the dual net (blue). that has one kind of vertex, edge and face, but two tiles that are regular polyhedra (tetrahedra and octahedra). The quasiregular net is illustrated in the same way as the regular nets in Fig. 7.

\section{Natural tilings, transitivity and duals}

The description of nets in terms of the tilings that carry them is a powerful tool for the systematic enumeration of structures (Delgado Friedrichs et al., 1999; O'Keeffe, 1999). We believe that it is also very useful for the systemization of properties of nets. For a given net, there are many tilings that carry that net; however, there is a natural tiling that we believe to be unique and which we now describe after some necessary definitions.

Firstly, we note that every net has a combinatorial symmetry which is the maximum symmetry possible for that net, and that usually an embedding as a sphere packing can be realized with that symmetry. There are exceptions to the possibility of realization as maximum-symmetry embeddings, which we consider elsewhere, but they will not concern us here. Secondly, we note that a tile will have faces that are cycles of
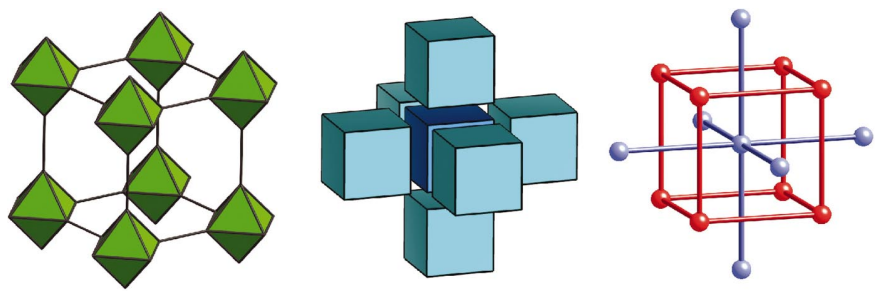

Figure 5

Left: the augmented primitive cubic lattice net (pcu). Center: the tiling. Right: the skeleton of one tile (red) with a fragment of the dual net (blue).
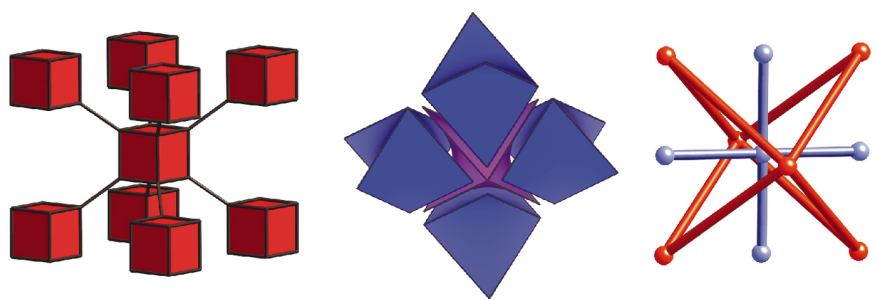

Figure 6

Left: the augmented body-centered cubic lattice net (bcu). Center: the tiling. Right: the skeleton of one tile (red) with a fragment of the dual nbo net (blue).
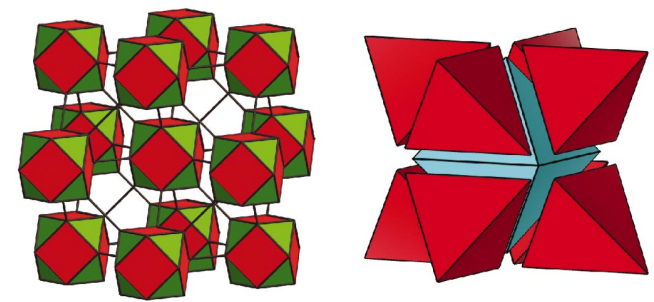

Figure 7

Left: the augmented face-centered cubic lattice net (fcu). Right: the tiling. 
the net. If we define a cycle sum of two cycles with one or more common edges in the usual way (Corey \& Petersson, 1972) as the set of edges not common to both cycles, then a ring may be defined as a cycle that is not the sum of two smaller cycles. A strong ring is defined (Goetzke \& Klein, 1991) as one that is not the sum of any number of smaller cycles (or, equivalently, smaller rings).

We now define a natural tiling as one that has the smallest possible tiles such that (i) the tiling has the maximum (combinatorial) symmetry and (ii) all the faces of the tiles are strong rings. There may be exceptional cases in which there is no natural tiling as defined above, or in which there is more than one natural tiling; again such instances do not concern us here. Indeed, in all the cases we consider, the natural tiling is readily apparent and unique.

We remark that the natural tiles are the natural cages (or holes) in the structure and their faces are the essential rings in the structure. There may be strong rings that are not faces of tiles; we give an example below ( $c f$. Fig. 8).

The tiles themselves are generalized polyhedra (cages) in which there may be vertices at which only two edges meet. They are conveniently described by face symbols $\left[M^{m} . N^{n} \ldots\right]$, which indicate that there are $m$ faces that are $M$-rings, $n$ faces that are $N$-rings etc. Notice the Euler equation relating the numbers of vertices $V$, edges $E$ and faces $F$, i.e. $F-E+V=2$ holds for cages.

In a tiling, specifically here a natural tiling, there will in general be $p$ kinds of vertex, $q$ kinds of edge, $r$ kinds of ring and $s$ kinds of tile. The transitivity (Delgado Friedrichs \& Huson, 2000) is defined as the array pqrs.
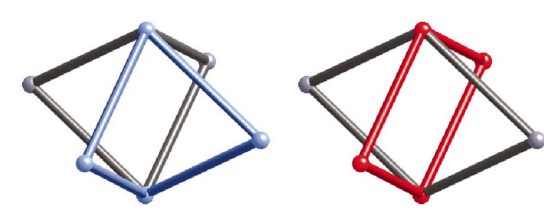

Figure 8

Skeletons of tiles for the bcu net. Left: a face ring (an essential 4-ring) is shown in blue. Right: an inessential 4-ring (not a face ring) is shown in red.

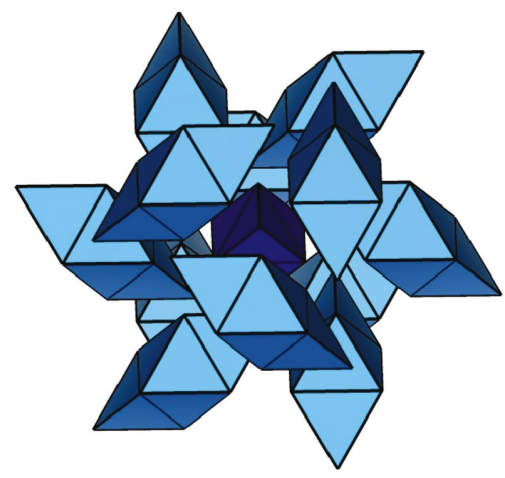

Figure 9

A tiling (not natural) for fcu that has one kind of vertex, one kind of edge, one kind of face and one kind of tile. Note that pairs of tiles have at most one face in common. The symmetry is $P a \overline{3}$.
The dual of a tiling is derived by placing a new vertex inside each tile and connecting pairs of new vertices that are in adjacent tiles by a new edge through the face common to the two tiles. Faces of the dual tiling are chosen in such a way that the dual of the dual is the original tiling; thus the dual tiling may not be the natural tiling of the net it carries (this complication does not arise in the cases we consider here). The transitivity of the dual of a tiling with transitivity pqrs is srqp. If a net has a unique natural tiling then the dual net is the net carried by the dual of that tiling. Intergrowth of a net and its dual in the case that both tilings are natural is of special interest; then all the edges of one net penetrate essential rings of the other, and all essential rings of one net are penetrated by edges of the other. In other words, all rings of one net are catenated with rings of the other net and we say that the nets are fully catenated.

Each edge of a net passes through a face of the dual tiling. If that face is an $N$-ring, $N$ of the original tiles meet at that edge. Similarly, each vertex is in the center of a dual tile; if that tile has $M$ vertices, $M$ of the original tiles meet at that vertex. The total number of edges of a dual tile is equal to the number of rings in the original net meeting at the vertex associated with that dual tile.

\section{Natural tilings, transitivity and duals of regular and quasiregular nets}

Natural tiles for the regular nets are shown in Figs. 2-6. In each case there is just one kind of tile with one kind of face so in each case the transitivity is 1111 . These are the only natural tilings we know of with transitivity 1111 , and it is pleasing that the regular nets correspond to the smallest possible transitivity (i.e. the array of smallest possible numbers for each of $p, q, r$, $s)$. However, we remark that we know of no proof that there are not other natural tilings with the same transitivity.

The tile for the srs net (Fig. 2) is a trihedron $\left[10^{3}\right]$ with 3 faces, 15 edges and 14 vertices and with symmetry 32 . The dual structure is the enantiomorph of the original net. In the symbolism of lattice complexes, ${ }^{+} Y^{*}$ and ${ }^{-} Y^{*}$ are a dual pair. The 10-rings are the only rings in the structure and they are all essential rings.

The tiles for the nbo net (Fig. 3) are octahedra $\left[6^{8}\right]$ with 18 vertices and 24 edges and with symmetry $m \overline{3} m$. Each tile has three 8-rings around a midsection but these are not strong
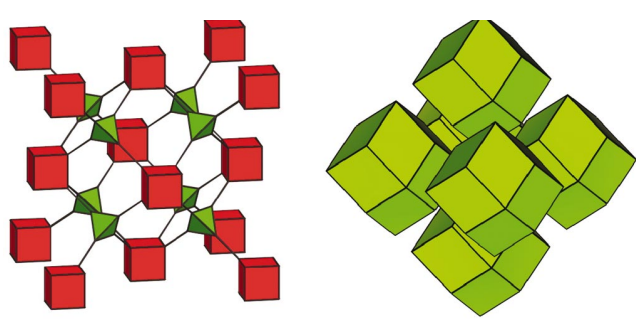

Figure 10

Left: the augmented fluorite net. Right: the tiling. 
rings as any one is the ring sum of four 6-rings. The dual is the 8-coordinated bcu structure.

The tiles for the dia net (Fig. 4 ) are tetrahedra $\left[6^{4}\right]$ with 10 vertices and 12 edges and with symmetry $\overline{4} 3 \mathrm{~m}$. The structure is self-dual.

The tiles for the pcu net (Fig. 5) are cubes [4 $\left.{ }^{6}\right]$ with 8 vertices and 12 edges and with symmetry $m \overline{3} m$. The structure is self-dual.

The tiles for the bcu net (Fig. 6) are tetrahedra $\left[4^{4}\right]$ with 6 vertices and 8 edges and with symmetry $4 / \mathrm{mmm}$. Each tile has two 4-rings around a midsection (Fig. 8). These are strong rings as there are no smaller rings (all the rings in the structure are 4-rings), but as they are not face rings they are not essential rings. It should be apparent from the figure that they could not serve as faces of tiles without breaking the symmetry, so they cannot serve as faces of natural tiles. The dual of bcu is the 4-coordinated nbo structure.

For the quasiregular net (fcu, Fig. 7), there are two kinds of tile $(s=2)$, namely a regular tetrahedron and a regular octahedron, but only one kind of face which is common to a tetrahedron and an octahedron. Accordingly, the transitivity is 1112. Again, we know of no other natural tiling with this transitivity and no proof that there are not others.

The quasiregular net allows an illustration of a tiling (Grünbaum \& Shephard, 1980) that is not a natural tiling, with transitivity 1111 . The tiles are obtained by gluing tetrahedra to two opposite faces of an octahedron and fitting these tiles together in a way (Fig. 9) that only one face is shared between a given pair of tiles. The symmetry of the tiling, $P a \overline{3}$, is less than the maximum symmetry of the net, $F m \dot{3} m$, and the tiles are not the smallest possible with faces that are strong rings, so the tiling is not natural. Notice that if we dissect the octahedra of the fcu net into two square pyramids we will again lose some symmetry and the square faces of the pyramids are not strong rings (they are the sums of the four triangular faces of the pyramids) so that tiling by tetrahedra and square pyramids would not be a natural tiling either.

The dual of the fcu net (transitivity 1112) must have two kinds of vertex and transitivity 2111 . It is in fact the net of the fluorite $\left(\mathrm{CaF}_{2}\right)$ structure for which our name is flu. Aspects of this structure are illustrated in Fig. 10 and some data are included in Table 1. Just as we believe that fcu is the only structure with transitivity 1112, so we believe that flu is the only one with transitivity 2111 . In this sense, the latter is the most regular binary structure. The tiling by rhombic dodecahedra is, of course, of great importance in many areas of solidstate physics and chemistry.

It is interesting that the property of self-duality is relatively uncommon among nets, yet it occurs in what are probably the most common nets observed (O'Keeffe et al., 2000) in crystal chemistry, i.e. the srs, dia and pcu nets, which are the most regular nets for triangular, tetrahedral and octahedral coordination. It is not surprising therefore to find two (or occasionally more) such nets intergrown when the basic net has low density (e.g. because of the presence of long links) and the occurrence of such pairs of intergrown nets is rather common (Batten \& Robson, 1998).

\section{Concluding remarks}

Our goal in this paper is to establish a basis for a systematic description of 3-periodic nets. We use an approach based on the concept of natural tilings to establish a transitivity pqrs. In this paper, we have described the structures $111 s$ (note that $s$ is necessarily equal to either 1 or 2 ). The next most regular structures have transitivity $11 r s$. These are also relatively few and generally important in crystal chemistry (an example is the net of the sodalite structure); we propose to call these 'semiregular' and to discuss them in the next paper of this series. We note that these semiregular structures have earlier been termed 'homogeneous' (Pearce, 1978) or 'quasiregular' (O'Keeffe \& Hyde, 1996).

Although we use tilings to assist in the description and classification of the nets, our main focus is on the regularity of coordination around the vertices. This is reflected in the order of symbols in the transitivity (vertices first, tiles last). If we were to focus on the tilings, a useful approach, which allows systematic enumeration, is through the use of Delaney symbols (Dress, 1985; Delgado Friedrichs et al., 1999). In this method, the tiles are divided into tetrahedral chambers with vertices at the center of the tile, the center of a face, the center of an edge and a vertex. The tiles in the tiling may be classified by the number of different chambers. The only tiling with just one kind of chamber is the tiling by cubes to produce the primitive cubic lattice; this is in accord with its identification with the only regular tiling. The tilings with nets that we have here called nbo, dia, bcu, fcu, flu are the only ones with just two kinds of chamber (Delgado Friedrichs, 1994). The only other structure we have described here, the regular 3-coordinated srs net, has a tiling with ten distinct chambers emphasizing that in classifying nets we should attach little weight to the symmetry of the tiles that form the natural tiling, and instead focus, as done here, on the vertices and their surroundings.

We remark that the tilings illustrated in this paper all appear in the remarkable, but insufficiently well known, book by Pearce (1978).

Work supported by the National Science Foundation grant DMR 0103036, and by the Donors of the Petroleum Research Fund, administered by the American Chemical Society.

\section{References}

Akporiaye, D. E. \& Price, G. D. (1989). Zeolites, 9, 23-32. Andries, K. J. \& Smith, J. V. (1994). Proc. R. Soc. London Ser. A, 444, 217-238.

Baerlocher, C., Meier, W. M. \& Olsen, D. H. (2001). Atlas of Zeolite Framework Types. Amsterdam: Elsevier. http://www.iza-structure. Org/databases/.

Barrer, R. M. \& Villiger, H. (1969). Z. Kristallogr. 128, 352-370.

Batten, S. R. \& Robson, R. (1998). Angew. Chem. Int. Ed. Engl. 37, 1461-1494.

Chung, S. J., Hahn, T. \& Klee, W. E. (1985). Acta Cryst. A40, 42-50. Corey, E. J. \& Petersson, G. A. (1972). J. Am. Chem. Soc. 94, 460-465. Coxeter, H. S. M. (1973). Regular Polytopes, 3rd ed. New York: Dover. 
Delgado Friedrichs, O. (1994). Doctoral thesis, University of Bielefeld, Germany.

Delgado Friedrichs, O., Dress, A. W. M., Huson, D. H., Klinowsky, J. \& Mackay, A. L. (1999). Nature (London), 400, 644-647.

Delgado Friedrichs, O. \& Huson, D. H. (2000). Discrete Comput. Geom. 24, 279-292.

Dress, A. W. M. (1985). Springer Lecture Notes in Mathematics, Vol. 1172, pp. 56-72. Berlin: Springer.

Euclid of Alexandria (ca 300 B.C.). Elements XIII.

Fischer, W. (1973). Z. Kristallogr. 138, 129-146.

Fischer, W. (1974). Z. Kristallogr. 140, 50-74.

Fischer, W. (1991a). Z. Kristallogr. 194, 67-85.

Fischer, W. (1991b). Z. Kristallogr. 194, 87-110.

Fischer, W. (1993). Z. Kristallogr. 205, 9-26.

Fischer, W. \& Koch, E. (1983). Lattice Complexes. In International Tables for Crystallography, Vol. A, edited by Th. Hahn. Dordrecht: Kluwer.

Goetzke, K. \& Klein, H.-J. (1991). J. Non-Cryst. Solids, 127, 215-220.

Grünbaum, B. \& Shephard, G. C. (1980). Bull. Am. Math. Soc. 3, 951-973.

Han, S. \& Smith, J. V. (1994). Acta Cryst. A50, 302-307.

Han, S. \& Smith, J. V. (1999a). Acta Cryst. A55, 332-341.

Han, S. \& Smith, J. V. (1999b). Acta Cryst. A55, 342-359.
Hyde, S. T. \& Ramsden, S. (2000). Chemical Topology, edited by D. Bonchev \& D. H. Rouvray, ch. 2. Amsterdam: Gordon and Breach.

Kepler, J. (1619). Harmonices Mundi. Linz.

Koch, E. \& Fischer, W. (1995). Z. Kristallogr. 210, 407-414.

O'Keeffe, M. (1991). Z. Kristallogr. 196, 21-37.

O'Keeffe, M. (1992). Acta Cryst. A48, 670-673.

O'Keeffe, M. (1995). Acta Cryst. A51, 916-920.

O'Keeffe, M. (1999). Nature (London), 400, 617-618.

O'Keeffe, M. \& Brese, N. E. (1992). Acta Cryst. A48, 663-669.

O'Keeffe, M., Eddaoudi, M., Li, H., Reineke, T. \& Yaghi, O. M. (2000). J. Solid State Chem. 152, 3-20.

O'Keeffe, M. \& Hyde, B. G. (1996). Crystal Structures I: Patterns and Symmetry. Washington: Mineral. Soc. Am.

Pearce, P. (1978). Structure in Nature is a Strategy for Design. Cambridge: MIT Press.

Smith, J. V. (1988). Chem. Rev. 88, 149-182.

Treacy, M. M. J., Randall, K. H., Rao, S., Perry, J. A. \& Chadi, D. J. (1997). Z. Kristallogr. 212, 768-791.

Wells, A. F. (1977). Three-Dimensional Nets and Polyhedra. New York: Wiley.

Wells, A. F. (1979). Further Studies of Three-Dimensional Nets. Am. Crystallogr. Assoc. Monogr. No. 8. 